\title{
Pediatric onco-hematological home care during COVID-19 pandemic
}

\author{
Matteo Amicucci ${ }^{1}$ - Federico Piccioni ${ }^{1}$ - Francesca Cocca ${ }^{1}$ - Emanuela Monteferrario ${ }^{1}$. Giuliana D'Elpidio ${ }^{2}$. \\ Luisa Tredici ${ }^{1}$ - Luciana Pellegrini ${ }^{1} \cdot$ Angela Mastronuzzi $^{1}$ - Andreea Cristina Schiopu ${ }^{3} \cdot$ Emanuela Tiozzo $^{4}$. \\ Immacolata Dall'Oglio ${ }^{4} \cdot$ Italo Ciaralli $^{1}$
}

Received: 12 April 2021 / Accepted: 10 September 2021 / Published online: 24 September 2021

(c) The Author(s), under exclusive licence to Springer-Verlag GmbH Germany, part of Springer Nature 2021

\begin{abstract}
The aim of the work is to describe the protocol adopted by the Home Care Service in pediatric onco-hematological patients of a large cancer institute in Italy during COVID-19 pandemic and to present preliminary data. Based on our experience, we have developed strategies to ensure continuity of care, non-abandonment, and protection of patients and operators in a period of emergency like this. In this context, the "COVID at home" protocol plays a central role. It aims to be able to safely manage COVID-19 positive onco-hematological patients, allowing nursing and medical care in the home setting, identifying patients at risk for COVID-19 infection, and rationalizing improper accesses to the hospital.
\end{abstract}

Keywords Pediatric Home Care $\cdot$ COVID-19 pandemic $\cdot$ Protocol

In Italy, Pediatric Home Care (PHC) can be a communitybased service (managed locally by the Health Service), provided to pediatric patients with long-term pathologies, or a hospital-based service (managed by the hospital), offered free of charge to patients in need of specialist care. This model is also described and analyzed in other international contests for pediatric patients with onco-hematological diseases $[1,2]$. In both cases, hospital professionals go directly to the patients' homes to provide treatments that are usually provided in the hospital, such as transfusions, antibiotic therapy infusions, fluid therapy, and oral and intravenous chemotherapy.

Our department offers updated treatments according to national and international protocols to pediatric patients

Matteo Amicucci

matteo.amicucci@opbg.net

1 Department of Onco Hematology and Cell and Gene Therapy, Bambino Gesù Children's Hospital, IRCCS, Sant'Onofrio Square, 4, 00165 Rome, Italy

2 Bambino Gesù Children's Hospital, IRCCS, University of Rome "Tor Vergata", Rome, Italy

3 Department of Paediatric Emergency, Bambino Gesù Children's Hospital, IRCCS, Rome, Italy

4 Professional Development, Continuing Education and Nursing Research Service, Bambino Gesù Children's Hospital, IRCCS, Rome, Italy suffering from oncological and hematological pathologies. Approximately 400 new patients with neoplasms of the blood and lymphatic system and solid tumors are diagnosed/treated every year. In 2017, our institute adopted a Home Care (HC) service (managed by the hospital), which can include pediatric onco-hematological patients who, after the acute phase, are able to be treated with the HC according to well-defined requirements. This service is guaranteed both at the patients' homes and at the various shelterhouses in the area. Thanks to this service, pediatric patients (1 month-18 years old) and young adults up to 25 years old under treatment at our institute are assisted, if they are domiciled farther than $30 \mathrm{~km}$ from the hospital. Assistance is provided for medical and nursing home visits: approximately 500 accesses per month and 60 patients assisted per week, of which about $5 \%$ are in an advanced stage of disease.

During the COVID-19 pandemic, there was much discussion on how to continue providing home care to our patients $[3,4]$. Much of the critical issues that emerged refer to shortcomings relating to territorial assistance which inevitably led to an overcrowding of hospitals. This is likely due to a difficulty in being able to reorganize local services such as home care in an emergency situation. Therefore, despite the difficulties, the goal is to describe how the home care service continued with complete safety thanks to having identified a specific management protocol. It was very important to identify patients "at risk" 


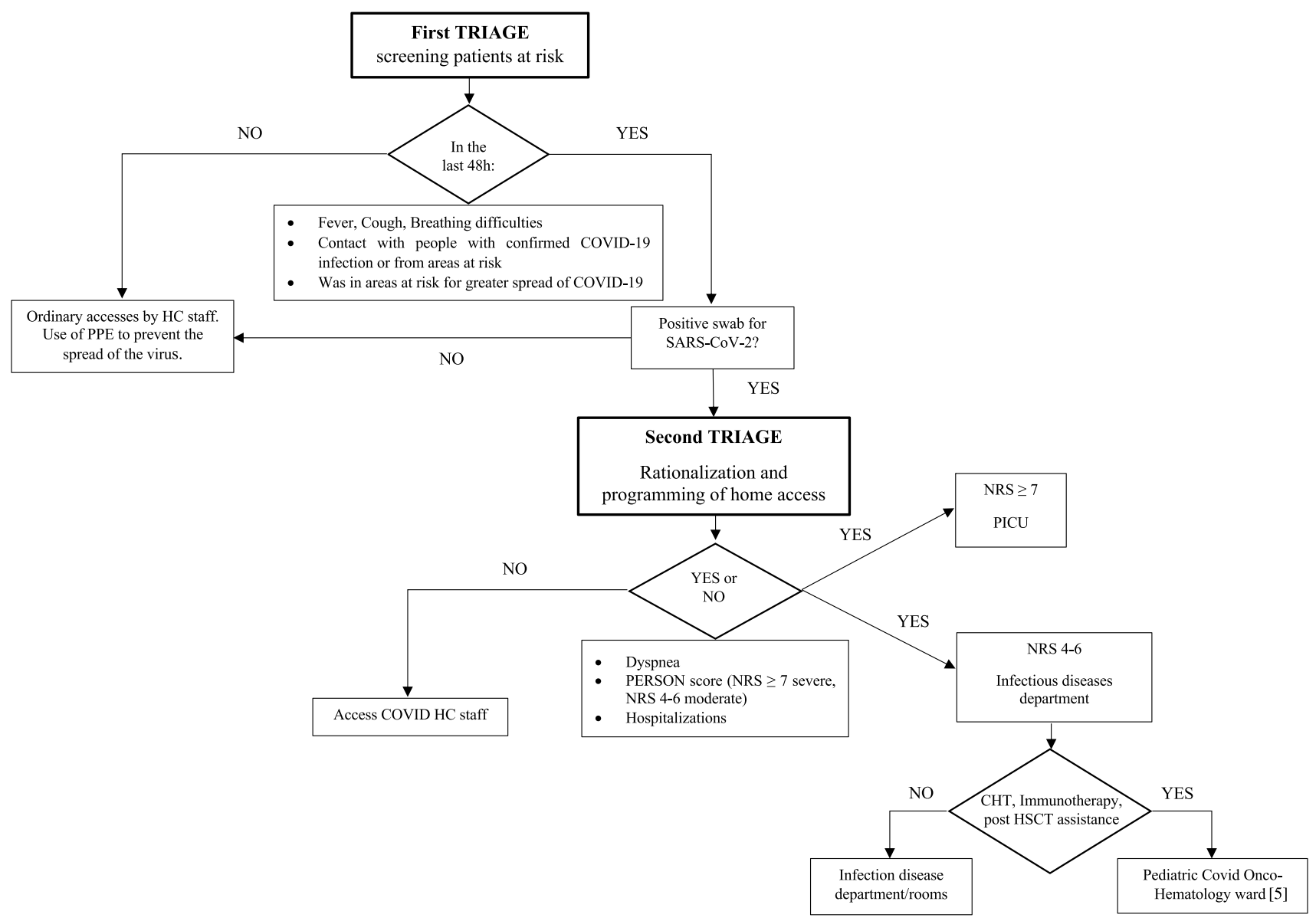

Fig. 1 Flow diagram to explain "Covid at home" protocol. Home Care (HC), Personal Protective Equipment (PPE), National Reporting System (NRS), Pediatric Intensive Care Unit, chemotherapy (CHT), hematopoietic stem cell transplant (HSCT)

for contagion of COVID-19 and to manage home access with well-defined criteria. The comparison between the operators made it possible to develop the "Covid at home" protocol. The protocol was created precisely to allow us to continue to provide medical and nursing assistance to pediatric onco-hematological patients as in the period prior to the COVID-19 pandemic.

Our protocol includes the following:

A first triage to all patients to identify patients at risk. The day before the scheduled home visit, a nurse contacts the patient by telephone and asks if in the last $48 \mathrm{~h}$ :

- he/she had fever, cough, and breathing difficulties.

- he/she had contact with people with confirmed COVID19 infection or from areas at risk.

- he/she was in areas at risk for greater spread of COVID19.

If the first triage was negative, the patient was managed following the ordinary $\mathrm{HC}$ accesses. In case of positivity at the first triage, the patient and the entire family were swabbed to ascertain positivity for SARS-CoV-2 and if positive, there were two treatment modalities.

If the patient was asymptomatic, the HC Covid team went to the patient's home to perform swabs, blood tests, transfusions, antibiotic or other therapy infusions, fluid therapy, chemotherapy, dressing changes, vital signs states, and medical visits according to the "Covid at home" protocol. If the patient was symptomatic, the medical staff proceeded with the evaluation based on the presence and severity of symptoms PERSONS score (Pain, Eating, Rehabilitation, Sleep, O2, Nausea/vomiting, and Suffering) and on the need for hospitalization as shown in the diagram in Fig. 1. The PERSONS score is a tool for symptom assessment and management in an oncological outpatient setting and the score is rated on a numerical rating scale (NRS) between 0 (no burden) and 10 (worst imaginable burden). If the patients were symptomatic with a NRS 4-6 (moderate) or NRS $\geq 7$ (severe) and needed hospitalization, they were hospitalized following specific protocols $[6,7]$.

At the second triage the patient is evaluated based on the presence and severity of symptoms and on the prognosis as 


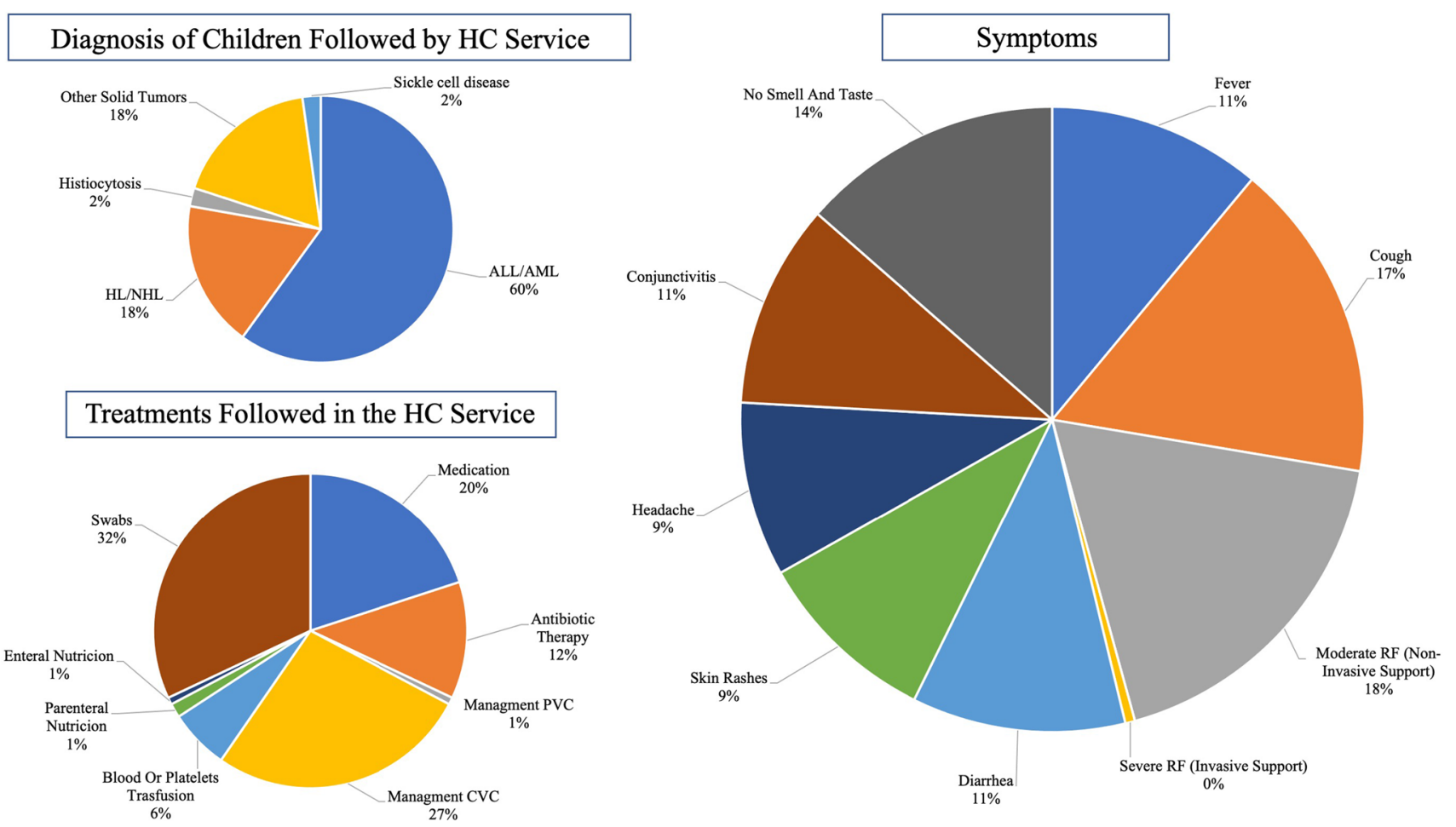

Fig. 2 Diagnosis, treatments, and symptomatology of COVID19-positive children managed in HC from March to December 2020. Home Care (HC), acute lymphoblastic leukemia (ALL), acute mye-

high, medium, or low risk. Based on these parameters, the doctor decides which accesses to perform. The patient is followed by the entire $\mathrm{HC}$ team without going to the hospital. High-risk patients are examined every day, medium-risk patients twice a week, and low-risk patients once a week. From March to May 2020, medical visits were always performed in the hospital or at patients' home. From June 1, medical staff have had the possibility to carry out the visit remotely or via a video call or teleconsultation. The team that provides assistance is equipped with all specific Personal Protective Equipment (PPE) and adequate training, transport, and disposal of infectious waste.

Detailed protocols were put into place for COVID-19 negative and positive patients in shelter-houses and residential facilities where home care was necessary. In this case, all these facilities were involved also in the management of local protocols.

Access was allowed only with a negative molecular swab and body temperature below $37^{\circ} \mathrm{C}$ for the patient and parents. It was mandatory to wear a surgical mask and perform regular hand disinfecting with specific precautions in the common areas (restricted access, alcoholic solution dispenser, and social distancing). For the management of loid leukemia (AML), Hodgkin's lymphoma (HL), non-Hodgkin's lymphoma (NHL), peripheral venous catheter (PVC), central venous catheter (CVC), respiratory failure $(\mathrm{RF})$

patients within these facilities, the staff followed the double triage protocol described above.

In 2020, in line with the previous year, approximately 500-600 accesses have been registered per month and 60-70 patients have been assisted per week, of which approximately $4 \%$ were in an advanced stage of disease. About $15 \%$ of the patients followed per week were COVID19 positive. A summary of the type of COVID-19 positive patients managed in $\mathrm{HC}$ is shown in Fig. 2.

It was not easy to carry out projects like these during the pandemic. As reinforced by a national survey, home care was kept operative and took place in all centers where it was already well structured in the area [8]. Otherwise, we believe that we would also have seen problems related to this type of management as well. Furthermore, our institute has always guaranteed the availability of resources and PPE. For this reason, we consider ourselves lucky. Our service has three medical vehicles at its disposal to carry out the at home services and continuous support from voluntary associations. We report this experience, although not supported by consistent data, in order to provide elements of comparison to colleagues who are facing situations similar to ours. 
Acknowledgements We would like to thank URP office to help carrying out the described project.

Author contribution Dr. AM, PF, and CI conceptualized and designed this commentary and drafted the initial manuscript, and DOI, CF, and ME reviewed and revised the manuscript. AM, DEG, TL, PL, MA, SAC, and TE approved the final manuscript as submitted and agree to be accountable for all aspects of the work.

Data availability Not applicable.

Code availability Not applicable.

\section{Declarations}

Ethics approval Not applicable.

Consent to participate Not applicable.

Consent for publication Not applicable.

Conflict of interest The authors declare no competing interests.

\section{References}

1. Hansson H, Hallström I, Kjaergaard $\mathrm{H}$ et al (2011) Hospital-based home care for children with cancer. Pediatr Blood Cancer 57:369377. https://doi.org/10.1002/pbc.23047

2. Parker G, Bhakta P, Lovett $\mathrm{C}$ et al (2006) Paediatric home care: a systematic review of randomized trials on costs and effectiveness.
J Health Serv Res Policy 11:110-119. https://doi.org/10.1258/ 135581906776318947

3. Porzio G, Cortellini A, Bruera E et al (2020) Home care for cancer patients during COVID-19 pandemic: the double triage protocol. J Pain Symptom Manage 60:e5-e7. https://doi.org/10.1016/j.jpain symman.2020.03.021

4. Varani S, Ostan R, Franchini L et al (2021) Caring advanced cancer patients at home during COVID-19 outbreak: burnout and psychological morbidity among palliative care professionals in Italy. $\mathrm{J}$ Pain Symptom Manage 61:e4-e12. https://doi.org/10.1016/j.jpain symman.2020.11.026

5. Amicucci M, Perigli F, D'Elpidio G, Ripà A, Mastronuzzi A, Ioris MAD, Schiopu AC, Dall'Oglio I, Ciaralli I (2021) A pediatric COVID hematology/oncology ward to guarantee adequate medical and nursing assistance. Pediatric Blood \& Cancer 68(8):e29113. https://doi.org/10.1002/pbc.29113

6. Cortellini A, Porzio G, Masel EK et al (2019) The PERSONS score for symptoms assessment in simultaneous care setting: a pilot study. Palliat Support Care 17:82-86. https://doi.org/10. 1017/S1478951518000238

7. Cortellini A, Porzio G, Cofini V et al (2020) The PERSONS score: a new tool for cancer patients' symptom assessment in simultaneous care and home care settings. Palliat Support Care 18:33-38. https://doi.org/10.1017/S1478951519000543

8. Amicucci M, Canesi M, Rostagno E et al (2020) How we have protected our patients: the Italian pediatric onco-hematology units' response to the COVID-19 pandemic. Pediatr Blood Cancer 67:e28505. https://doi.org/10.1002/pbc.28505

Publisher's note Springer Nature remains neutral with regard to jurisdictional claims in published maps and institutional affiliations. 